\title{
Molecular markers for diagnostic cytology of neoplasms in the head region of the pancreas: mutation of K-ras and overexpression of the $\mathrm{p} 53$ protein product
}

\author{
J M van Es, M M Polak, F M van den Berg, T B Ramsoekh, M E Craanen, \\ R H Hruban, G J A Offerhaus
}

\begin{abstract}
Aims-To determine the potential efficiency of molecular markers specific for neoplastic change-mutations of the K-ras oncogene and the p53 tumour suppressor gene-in diagnosing pancreatic carcinoma.

Methods-Archival cytology samples obtained from 17 patients with established pancreatic carcinoma were assayed for alterations in K-ras and p53. To detect changes in p53 expression, immunocytochemistry with polyclonal antibody CM1 was performed on the archival cytology slides after destaining. Mutations in $K$-ras codon 12 were then analysed on the scrapings of the same slides using mutant enriched polymerase chain reaction (PCR) amplification and restriction fragment length polymorphism analysis with allele specific oligonucleotide hybridisation for confirmation and characterisation.
\end{abstract}

Results-False negative results were recorded for five of the cytology slides when compared with p53 immunostaining of the surgical resection specimen. These five cases had been stained previously with Giemsa which interacts adversely with the immunostaining in contrast to the Papanicolaou procedure. The K-ras codon 12 mutations followed the well established distribution frequency and spectrum for pancreatic cancer and corresponded with the findings in the resection specimens in all cases. Two scrapings yielded insufficient DNA for PCR. Importantly, for two cases with an inconclusive cytology diagnosis on routine light microscopy, the diagnosis was confirmed by one of the molecular markers. The application of the molecular markers increased the diagnostic accuracy of cytology in this small study from 76 to $89 \%$.

Conclusions-The study indicates that assessment of alterations in the $K$-ras and p53 genes may be a valuable adjunct to diagnostic cytopathology of the head region of the pancreas, although there are some difficulties which will have to be overcome.

(f Clin Pathol 1995;48:218-222)

Keywords: K-ras, p53, polymerase chain reaction, pancreatic cancer.

Pancreatic cancer is a major contributor to cancer related death in the West. ${ }^{1}$ Most patients with pancreatic cancer present with obstructive jaundice, abdominal discomfort and weight loss, ${ }^{1}$ and are usually evaluated by endoscopic retrograde cholangiopancreatography (ERCP) and computed tomography (CT). Cytology specimens can be collected during the above procedures, either by brush cytology or fine needle aspiration. ${ }^{12}$ Although these cytology specimens can often be used to establish a definitive diagnosis of cancer, it may be difficult to distinguish between reactive and neoplastic changes. In about $30 \%$ of cases it is impossible to reach a definitive diagnosis. ${ }^{1}$

Molecular markers specific for neoplastic disease could potentially improve the diagnostic yield of cytology and thus avoid unnecessary delay in treating patients. Mutations in the Kras oncogene and the p53 tumour suppressor gene are two such potential markers for pancreatic cancer. ${ }^{34} \mathrm{~K}$-ras oncogene mutations occur in 80 to $90 \%$ of pancreatic cancinomas and are equally prevalent in cancers of the head, body and tail of the pancreas. ${ }^{56}$ These mutations may occur relatively early in the development of pancreatic cancer, ${ }^{7}$ detection of which could improve prognosis. K-ras gene mutations are usually limited to one codon and therefore can be detected using a small number of probes. ${ }^{6}$ Recently developed enrichment techniques can be used to detect rare mutant cells in small samples such as cytology specimens. $^{67}$

In contrast to $\mathrm{K}$-ras mutations, p53 tumour suppressor gene mutations occur at multiple sites, mostly in exons 5 to $8,{ }^{8}$ making mutational analysis of p53 more difficult. A feature that facilitates the study and potential use of p53 as a molecular marker is the stability of the mutant p53 protein product, which can be detected immunohistochemically. ${ }^{9-11}$ p53 gene 

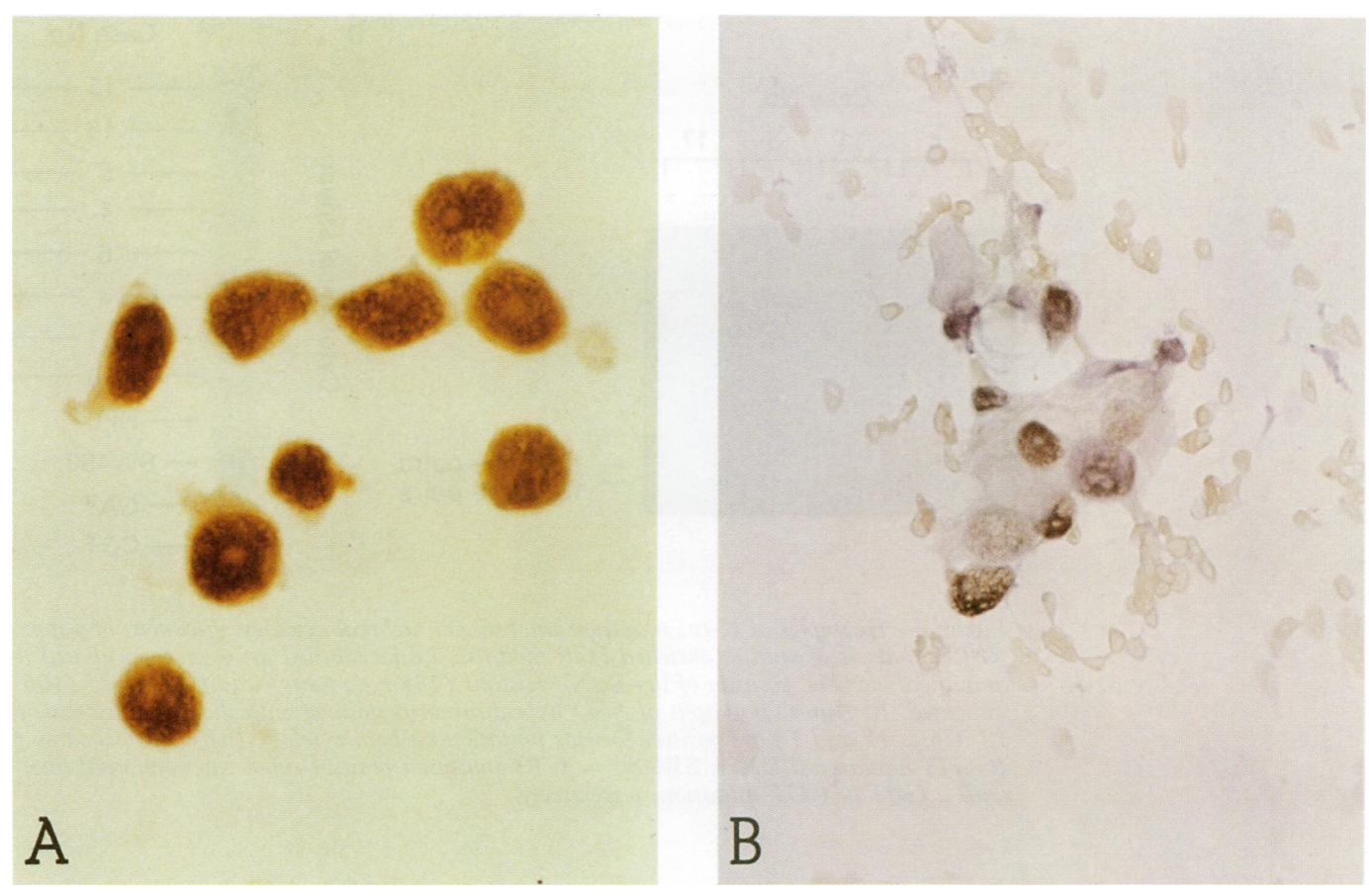

Figure 1 Immunocytochemistry of representative cell specimens following PAP staining. A: Human colon cancer cell line SW480; B: cytological specimen from case 8. p53 overexpression can be seen as brown nuclear staining.

mutations are present in 50 to $70 \%$ of pancreatic carcinomas, and almost exclusively in invasive tumours. In contrast to K-ras mutations, mutant p53 is seldom found in in situ lesions. ${ }^{12}$ This suggests that changes in the p53 gene occur late in carcinogenesis and therefore p53 is a relatively specific marker for malignancy.

\section{Methods}

Surgical and cytology specimens from 17 patients (nine men and eight women; mean age 60 years, range $40-80$ years) with adenocarcinoma of the pancreas were retrospectively analysed. Inclusion criteria comprised a definitive diagnosis based on examination of a surgical resection specimen, at least two qualitatively good cytology glass slides collected prior to the final diagnosis (one for this study and the other to be archived), and a diagnosis of adenocarcinoma of the head region of the pancreas. Seven of the cytology specimens had been air-dried, fixed in methanol and stained with Giemsa. The remaining 10 specimens had been fixed in $100 \%$ ethanol and stained according to the Papanicolaou (PAP) procedure. All histological material had been routinely fixed in $4 \%$ phosphate buffered formalin and embedded in paraffin wax. Sections $(5 \mu \mathrm{m})$ were mounted on organosilane coated glass slides and dried overnight at $56^{\circ} \mathrm{C}$. The anti-p53 polyclonal antibody $\mathrm{CM} 1$ was used for immunochemistry (Novocastra Laboratories, Newcastle upon Tyne, UK). The surgical specimens were stained for $\mathrm{p} 53$ (over)expression by immunohistochemistry using target unmasking fluid as described previously. ${ }^{12}$ Prior to the study, immunocytochemistry and the polymerase chain reaction/restriction fragment

Results of p53 immunocytochemistry and PCR-RFLP analysis with subsequent ASO hybridisation for K-ras codon 12 mutations in cytology samples obtained from 17 patients with pancreatic carcinoma

\begin{tabular}{|c|c|c|c|c|c|c|c|}
\hline \multirow[b]{2}{*}{ Case No. } & \multicolumn{2}{|c|}{ p53 Immunostaining } & \multicolumn{2}{|c|}{$P C R$ mutation } & \multicolumn{2}{|c|}{ ASO hybrydisation } & \multirow[b]{2}{*}{ New amino acid } \\
\hline & Surgical & Cytologial & Surgical & Cytological & Surgical & Cytological & \\
\hline 1 & - & $-^{*}$ & - & - & - & - & \\
\hline 2 & + & $+^{*}$ & - & no DNA & - & & \\
\hline 3 & + & + & ++ & ++ & GAT & GAT & ASP \\
\hline 4 & + & $-{ }^{*}$ & ++ & ++ & GAT & GAT & ASP \\
\hline 5 & + & $-^{*}$ & + & + & CGT & CGT & ARG \\
\hline 6 & - & - & ++ & ++ & GAT & GAT & ASP \\
\hline 7 & - & - & - & - & - & - & \\
\hline 8 & + & + & - & - & - & - & \\
\hline 9 & + & + & - & - & - & - & \\
\hline 10 & - & - & + & ++ & GAT & GAT & ASP \\
\hline 11 & + & $-^{*}$ & ++ & ++ & GTT & GTT & VAL \\
\hline 12 & + & + & ++ & no DNA & GAT & & ASP \\
\hline 13 & + & $-^{*}$ & - & - & - & - & \\
\hline 14 & - & - & + & ++ & GTT & GTT & VAL \\
\hline 15 & + & + & ++ & ++ & GAT & GAT & ASP \\
\hline 16 & + & $-{ }^{*}$ & + & ++ & GTT & GTT & VAL \\
\hline 17 & - & - & - & - & - & - & \\
\hline
\end{tabular}

* Cytology samples stained with Giemsa.

Mutant and wild-type on gel ( + ); only mutant band in RFLP $(++)$. 

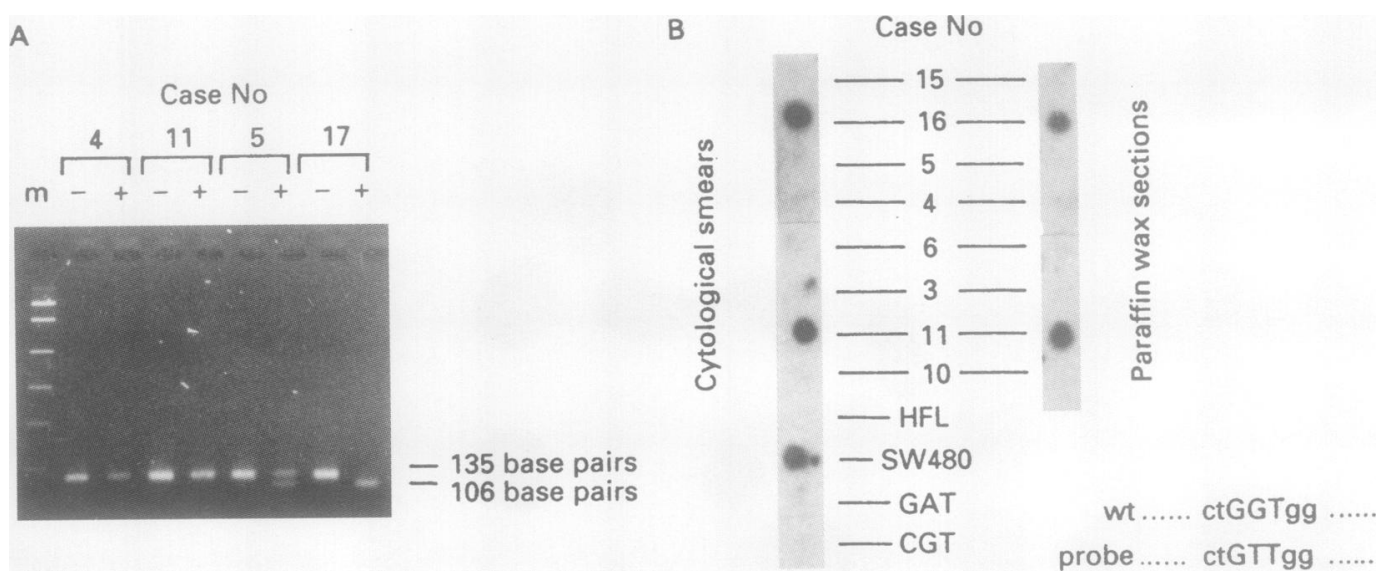

Figure 2 Examples of $K$-ras mutation analysis on archival cytology specimens of patients with pancreatic cancer. A: RFLP analysis of mutant enriched PCR products. Lanes labelled as + were incubated with BstNl after a second PCR amplification. The position of the Bst Nl resistant (135 base pairs) and the cleaved (106 base pairs) products are indicated. B: Autoradiographs of ASO hybridisation performed with the oligonucleotide probe containing GTT at codon 12. Cases 11 and 16 are positive for this mutation on both cytology (left) and histology (right). HFL = wild-type (GGT) human cell DNA;SW480 = GTT mutation positive colon carcinoma cell line; GAT/CGT = control samples with a $G A T$ or $C G T$ mutation, respectively.

length polymorphism analysis (PCR-RFLP) were assessed and developed on the human colon cancer cell line SW480, ${ }^{12-14}$ with known $\mathrm{K}$-ras and p53 mutations (fig 1). This also enabled us to determine the influence of Giemsa or PAP staining.

Based on the pattern of staining seen in the cell line, we developed a modified immunostaining procedure to optimise staining of the cytology specimens. Briefly, after dehydration in graded ethanol, the specimens were rinsed twice in distilled water and then fixed for 10 minutes in $4 \%$ paraformaldehyde in phosphate buffered saline (PBS). The specimens were then rinsed three times in distilled water and processed as described before for p53 immunocytochemistry. ${ }^{101215}$

After detection of p53 expression by immunocytochemistry and assessment of staining (nuclear positivity), the scrapings of the same glass slides were used for DNA isolation and PCR, as described previously. ${ }^{16}$

$\mathrm{K}$-ras mutations were determined in both histological and cytological material by a combination of mutant enriched PCR-RFLP analysis and allele specific oligonucleotide (ASO) hybridisation. ${ }^{6}$ Briefly, this technique uses a mismatched $5^{\prime}$ primer which generates a BstN1 restriction site at $\mathrm{K}$-ras codon 12 , such that digestion of the PCR product with BstN1 cleaves wild-type sequences and leaves amplified mutant alleles intact. Mutations are then verified and typed by ASO hybridisation.

In this study a cytology test was regarded as positive when either $\mathrm{p} 53$ immunocytochemistry or K-ras PCR-RFLP analysis with subsequent ASO hybridisation was positive. Efficacy was defined as the percentage of tumours correctly classified by the presence or absence of one of these molecular markers. ${ }^{10}$

\section{Results}

The results of the study are summarised in the table. Overexpression of p53 was detected in six of 17 (35\%) cytology specimens (fig 1). By contrast, p53 overexpression was observed in $11(66 \%)$ of the 17 surgical specimens; in five cases $\mathrm{p} 53$ positivity observed on histology was not confirmed on cytology.

Resistance to BstN1 digestion was observed in $10(60 \%)$ of the 17 cytology specimens (fig 2); scraping yielded insufficient DNA for PCR amplification in two cases. All cases with a positive result on PCR-RFLP analysis of the cytology specimens were also positive on histological analysis. In the five negative cytology cases PCR-RFLP analysis was also negative for the surgical specimens. Mutation was confirmed by ASO hybridisation in all positive cytology specimens. No discrepancies were found when ASO hybridisation was performed on the surgical and cytology specimens from the same patient. Mutations identified included guanine to adenine transitions (six cases), guanine to thymine transversions (three cases) and one guanine to cytosine transversion.

Based solely on either one of the cytological molecular markers, a diagnosis of pancreatic carcinoma would have been missed in four of $17(24 \%)$ cases, resulting in an efficacy of $76 \%$.

Four of the 17 cytology slides were read as negative or inconclusive on routine light microscopy; two of these were positive on either p53 immunocytochemistry or K-ras mutation analysis. This increased the diagnostic accuracy from 76 to $89 \%$.

\section{Discussion}

$\mathrm{K}$-ras and p53 gene mutations are believed to play a critical role in the development of invasive carcinoma of the pancreas. Detection of K-ras gene mutation provides an attractive molecular genetic probe for distinguishing between reactive and neoplastic changes in cytological samples. This is because K-ras mutations are present in most (80 to $90 \%$ ) pancreatic carcinomas, because they occur early in 
the development of pancreatic cancer, rendering early detection possible, and because they are limited to one codon and therefore detectable using a limited number of probes and primers. ${ }^{35-7}$ Mutations of the p53 tumour suppressor gene are similarly attractive because these mutations lead to stabilisation and therefore apparent overexpression of the $\mathrm{p} 53$ protein product, which can be readily detected using immunocytochemistry. Furthermore, p53 mutations occur exclusively in in situ or invasive carcinoma of the pancreas. ${ }^{4713}$

The present study indicates that PCR followed by RFLP analysis and immunohistochemistry can be used to detect K-ras and p53 point mutations, respectively, in cytology specimens and suggests that these markers may be of use in the diagnosis of pancreatic cancer, confirming previous reports. ${ }^{17-20}$ These techniques, when applied to archival tissue, were not without some technical problems. Of the 17 carcinomas, only six (35\%) showed p53 overexpression on cytology and in five cases this result was not confirmed by histology. These five cases were stained with Giemsa which appears to interact adversely with the p53 immunostaining procedure. In the colon cancer cell line SW480 we were unable to achieve reliable p53 immunostaining following Giemsa staining, whereas immunocytochemistry performed on cytological specimens stained using the PAP method gave excellent results. In addition, one discordant sample (case 13) was a sampling error since no neoplastic tumour cells were detected in this specimen on routine cytological evaluation.

Technical procedures such as fixation are critical for optimal p53 immunocytochemistry and in that regard the retrospective nature of this study has important drawbacks. In prospective studies preparation of separate samples for p53 immunocytochemistry, which could then be used for DNA isolation and PCR, may be advisable.

K-ras mutations were detected in 10 of 17 $(59 \%)$ cases. There was no discrepancy between the results obtained on histology and cytology suggesting that immunocytochemistry performed on cytological material even after routine fixation and staining has no adverse effects on subsequent PCR amplification and ASO hybridisation. The frequency distribution of the specific mutations-glycine to aspartic acid in $60 \%$, glycine to valine in $30 \%$ and glycine to arginine in $10 \%$-is in good agreement with the results obtained in our previous ${ }^{6}$ and in other studies. ${ }^{35}$ Interestingly, all positive results on PCR-RFLP analysis were confirmed by ASO hybridisation. This indicates that PCRRFLP analysis is a sufficiently reliable methodology for the determination of the presence or absence of mutations when there is no need to specify the exact type of mutation present. As PCR-RFLP analysis is a relatively simple and straightforward technique, this would be the preferred methodology for routine screening of mutations in cytological samples or in pancreatic juice. ${ }^{1718}$ ASO hybridisation can be used for confirmation of the diagnosis in borderline cases.

K-ras or p53 mutations are indicative of malignancy. On combining these tumour markers, 13 of the 17 cases were positive, yielding $76 \%$ efficacy. As an adjunct to routine procedures this could certainly increase the diagnostic yield of pancreatic cytopathology. The rate of $\mathrm{p} 53$ overexpression and K-ras mutation in non-neoplastic pancreatic specimens needs to be explored in larger series of patients.

Two cases in this small study illustrate the potential use of the diagnostic application of p53 and K-ras assays as a supplemental technique in diagnostic cytology. In one case, in which the material was inconclusively diagnosed as "atypical cells suspicious for malignancy" (case 8), the demonstration of p53 overexpression confirmed malignancy. In the second case (case 14), the initial diagnosis of "atypical cells", either reactive or neoplastic, was complemented by the unequivocal presence of a K-ras mutation.

In summary, while there are important technical caveats, this study indicates that molecular and immunocytochemical techniques for detecting $\mathrm{K}$-ras and p53 mutations are feasible and that detection of these molecular markers may be a valuable adjunct to diagnostic cytopathology.

1 Warsaw AL, Fernandez-del-Castillo C. Pancreatic carcinoma. N Engl F Med 1992;326:455-65.

2 Huibregtse K. Endoscopic biliary and pancreatic drainage. Stuttgart: Georg Thieme Verlag, 1988

3 Almoquera C, Shibata D, Forrester K, Martin J, Arnheim N, Perucho M. Most human carcinomas of the exocrine pancreas contain mutant c-K-ras genes. Cell 1988;53 549-54

4 Barton CM, Stoddon SL, Hughes CM. Abnormalities of the p53 tumorsupressor gene in human pancreatic cancer. Br f Cancer 1991;64:1076-82.

5 Bos JL. ras Oncogenes in human cancer. Cancer Res 1989; 49:4682-9.

6 Hruban RH, Mansfeld van ADM, Offerhaus GJA, van Weering DHJ, Allison DC, Goodman SN, et al. K-ras mutations in adenocarcinoma of the pancreas. Am 7 Pathol 1993;143:545-54

7 DiGiuseppe JA, Hruban RH, Offerhaus GJA, Clement MJ, van den Beig F, Cameron JL, et al. K-ras mutations in mucinous pancreatic ductal hyperplasia from a patient with a family history of pancreatic carcinoma. Am $\mathfrak{F}$ Pathol 1994;144:889-95.

8 Hollstein M, Sidranski D, Vogelstein B, Harris CC. p53 mutations in human cancers. Science 1991;253:49-53.

9 Levine AJ, Momand J, Finaly CA. The p53 tumor supressor gene. Nature 1991;351:453-6.

10 Baas IO, Mulder JWR, Offerhaus GJA, Vogelstein B, Hamilton SR. An evaluation of six antibodies for immunohistochemistry of mutant p53 gene product in archival histochemistry of mutant p53 gene product
colorectal neoplasms. $\mathcal{F}$ Pathol $1994 ; 172: 5-12$.

11 Hall PA, Lane DP. p53 in tumour pathology: can we trust immunohistochemistry?. . Revisited! f Pathol 1994;172: immu.

12 van den Berg FM, Polak M, Baas IO, Offerhaus GJA. Detection of $\mathrm{p} 53$ overexpression in routinely paraffinembedded tissue of human carcinomas using a novel targe unmasking fluid. Am $\mathcal{F}$ Pathol 1993;142:381-5.

13 DiGuiseppe JA, Hruban RH, Goodman SN, Polak M, van den Berg FM, Allison DC, et al. Overexpression of the p53 tumor suppressor protein in pancreatic carcinoma. Am f Clin Pathol 1994;101:684-8.

14 Verlaan-de Vries $M$, Bogaard ME, Elst van den $\mathrm{H}$, Boom van $\mathrm{JH}, \mathrm{Eb}$ van der AJ, Bos JL. A dot-blot screening procedure for mutated ras oncogenes using synthetic oligodeoxynucleotides. Gene 1986;50:313-20.

15 van den Berg FM, Tigges AJ, Schipper MEI, Den HartogJager FCA, Kroes WGM, Walboomers JMM. Expression Jager FCA, Kroes WGM, Wal ooomers JMM. Expression
of the nuclear oncogene p 53 in colon tumours. $\mathcal{f}$ Pathol of the nuclear on

16 Smits HL, Tieben LM, Tjong-A-Hung SP, Jebbink MF, Minnaar RP, Jansen CL, et al. Detection and typing of HPV present in fixed and stained archival cervical smears by a polymerase chain reaction and direct sequence analysis allows the identification of a broad spectrum of HPVs. 17 Shibata D, Almoquera C, Forrester K, Dunitz J, Martin 
SE, Cosgrove MM, et al. Detection of c-K-ras mutations in fine needle aspirate from human pancreatic adenocarcinomas. Cancer Res 1990;50:1279-83.

18 Tada M, Omata M, Kawai S, Saisho H, Ohto M, Saiki RK, et al. Detection of ras gene mutations in pancreatic juice and peripheral blood of patients with pancreatic adenocarcinoma. Cancer Res 1993;53:2472-4.
19 Hall PA, Ray A, Lemoine NR, Midgley CA, Krausz T, Lane D. p53 immunostaining as a marker of malignant disease in diagnostic cytopathology. Lancet 1991;338:513-14. 20 Dowell SP, Philip OGW, Nawal WD, Lane DP, Hall PA. Retrospective assessment of the clinical utility of immunocytochemical detection of $\mathrm{p} 53$ protein in cytological specimens [abstract]. I Pathol 1994;172(Suppl): 132A. 